\title{
Identification of Chalcogen Point-Defect Sites in Silicon by Total-Energy Calculations
}

\author{
Franz Beeler \\ Max-Planck-Institut für Festkörperforschung, D-7000 Stuttgart 80, Federal Republic of Germany \\ and \\ Matthias Scheffler \\ Max-Planck-Institut für Festkörperforschung, D-7000 Stuttgart 80, Federal Republic of Germany, \\ and Physikalisch-Technische Bundesanstalt, D-3300 Braunschweig, Federal Republic of Germany \\ and \\ Ove Jepsen and Olle Gunnarsson \\ Max-Planck-Institut für Festkörperforschung, D-7000 Stuttgart 80, Federal Republic of Germany \\ (Received 12 February 1985)
}

\begin{abstract}
We report parameter-free, self-consistent, electronic-structure and total-energy calculations for chalcogen point defects in crystalline silicon. Both possible defect sites of tetrahedral symmetry are investigated. The calculated total energies are discussed in terms of dissolution and reaction energies. From these, the stable defect position is identified as substitutional. The comparison of our theoretical results (spin and ground-state wave function) with experimental data such as infraredabsorption, EPR, and ENDOR spectra further supports this identification.
\end{abstract}

PACS numbers: $61.70 . \mathrm{Rj}, 66.30 . \mathrm{Jt}, 71.45 . \mathrm{Nt}$

Impurities in semiconductors generally exist in several different configurations, such as interstitials, substitutional sites, and complexes. ${ }^{1}$ Their stabilities and relative concentrations are primarily determined by the different dissolution energies. So far, little is known about these energies for different impurities and even for a particular impurity on different sites. Therefore, previous identifications of the geometrical configurations of defects were based on a comparison of theoretical electronic-structure calculations with experimentally observed quantities, such as defect wave-function symmetries (e.g., Ludwig, ${ }^{2}$ and Meyer, Spaeth, and $\left.S_{\text {cheffler }}{ }^{3}\right)$. In this paper we show that total-energy calculations can be used to determine the impurity site.

The stable configuration of chalcogen point-defect impurities in silicon has for a long time been unclear. Extensive experimental investigations have been performed on these centers, ${ }^{4-14}$ partly out of technological interest (e.g., infrared detectors and solar cells).4,10 Much experimental work was devoted to infrared absorption studies (see Refs. 4, 5, and references therein) which allow the determination of the energetic positions and symmetries of the ground state and various (effective-mass-like) excited states. The spectra could be interpreted in a one-electron picture. From an analysis of dipole selection rules, the symmetry of the deep-level wave function was determined to belong to the $A_{1}$ representation of the $T_{d}$ point group. This result was confirmed by electron paramagnetic resonance (EPR) and electron-nuclear doubleresonance (ENDOR) experiments. ${ }^{2,6,9}$ Furthermore, deep-level energies were determined by deep-level transient spectroscopy ${ }^{7,8}$ and Hall-effect measurements. ${ }^{11,12}$ Despite this extensive experimental work it has not been possible to identify whether isolated chalcogen impurities in Si occupy substitutional or $T_{d}$ interstitial sites. Many authors suggested that $\mathrm{S}, \mathrm{Se}$, and Te atoms are incorporated substitutionally, $2,4,9$ but the possibility of the interstitial site was also mentioned frequently. ${ }^{2,4,6,14}$ Recently, Niklas and Spaeth ${ }^{6}$ performed detailed ENDOR experiments on $\mathrm{Si}$ :Te and $\mathrm{Si}:$ Se. Their analysis of the spin densities at many shells of Si neighbors led them to suggest that the interstitial position appears to be the more likely site. In this paper we report self-consistent total-energy calculations for $\mathrm{S}$, Se, and Te impurities in silicon. We show that total-energy calculations can clearly distinguish between the above-mentioned alternative sites. The theoretical results identify the stable site of $\mathrm{S}, \mathrm{Se}$, and Te point defects in silicon as substitutional.

Green's-function methods have been shown to be powerful for electron-structure calculations of localized defects. ${ }^{15,16}$ Recently these methods have been developed to a state which also allows one to evaluate the total energy of the many-electron system ${ }^{17-20}$ and its gradient, i.e., the forces. ${ }^{21}$ Here we use the linearmuffin-tin-orbital (LMTO) Green's-function method in the atomic-spheres approximation. ${ }^{17}$ We use a variational expression (within the atomic-spheres approximation) for the total energy, which therefore converges faster than the eigenvalue spectrum. Further details will be discussed in a subsequent paper. ${ }^{22}$ The exchange-correlation energy is treated in the localdensity approximation (LDA). It is well-known that as a result of this approximation and the neglect of the 
discontinuity of the exchange-correlation functional derivative, ${ }^{23,24}$ the band gap in $\mathrm{Si}$ is about half of the experimental value. The results presented here were calculated with this too-small gap. In order to check the sensitivity of the results to the gap size we have performed the following tests: (1) The perfect-crystal Green's function was calculated from a band structure with the conduction band shifted rigidly to give the experimental gap, i.e., using the so-called "scissor" operator. $^{25}$ (2) The potential was modified so that the band-structure calculation gave a virtually unchanged valence band but the correct band gap. The three approaches-i.e., the pure LDA and methods (1) and (2) - gave different total energies which in the worst case differed by as much as $3.6 \mathrm{eV} .^{26}$ Thus, the uncertainty of total energies due to the band-gap problem can indeed be significant. This is a general problem of the LDA and not due to a Green's-function method. Since it is not clear which treatment of the problem is preferable we show in this paper only the pure (not modified) LDA results. We emphasize that the conclusions presented below are exactly the same for all three methods despite the above-mentioned uncertainty.

To discuss the stability of the two possible impurity configurations of $T_{d}$ symmetry, we first calculated the total energies of the processes in which chalcogens diffuse from the gas phase into a Si crystal and occupy a substitutional (sub) or $T_{d}$-interstitial (int) site. In both cases the initial state is an isolated chalcogen atom and a perfect $\mathrm{Si}$ crystal. The final state for the reaction "sub" is a Si crystal where a chalcogen atom replaces a $\mathrm{Si}$ atom, which has been moved to the surface. The differences between the calculated total energies of the final and initial states (dissolution energies) are given in Table I. In this paper we assume that the $\mathrm{Si}$ crystal is undoped. A discussion of the dissolution- and reaction-energy dependences on the Fermi-level position is given elsewhere. The energies in Table I refer to the neutral situation. For the substitutional centers these energies are obtained by calculation of the double-positive charge state and addition of two electrons to the conduction band. As men-

TABLE I. Calculated total energies in electronvolts (Ref. 27) for various processes of neutral chalcogens in silicon (see text).

\begin{tabular}{lccr}
\hline \hline & $\mathrm{S}^{0}$ & $\mathrm{Se}^{0}$ & $\mathrm{Te}^{0}$ \\
\hline$E^{\text {sub }}$ & -3.8 & -3.2 & -1.9 \\
$E^{\text {int }}$ & -0.1 & +3.2 & +9.1 \\
$E^{\text {iic }}$ & +0.3 & +3.0 & +7.6 \\
\hline \hline
\end{tabular}

tioned above, we use the pure LDA results, i.e., a theoretical band gap of $0.5 \mathrm{eV}$. The results in Table I show that the substitutional incorporation is exothermic for all chalcogens, and that this process is energetically clearly favored compared to the interstitial incorporation. Further evidence for the stability of the substitutional lattice site comes from considering the process where a substitutional chalcogen and a $\mathrm{Si} T_{d}$ selfinterstitial change places. This process is called the interstitial-interchange reaction (labeled iic in Table I). The results show that at nonzero temperature the relative occupancy of interstitial and substitutional sites depends sensitively on the atomic number. The stability of the substitutional site relative to the interstitial site increases from $\mathrm{S}$ to $\mathrm{Se}$ to $\mathrm{Te}$. From the last line of Table I it follows that at nonzero temperature a certain amount of $\mathrm{S}$ atoms may exist as interstitials if $\mathrm{Si}$ selfinterstitials are present. The accuracy of the calculation, in particular the uncertainty due to the band-gap problem, does not allow us to decide if the iic process for $S$ is really slightly endothermic, or if it is slightly exothermic. Either way, the theoretical results suggest that the diffusion of sulfur is partly determined by an interstitial component, whereas this is significantly less important for $\mathrm{Se}$ and $\mathrm{Te}$. It is interesting to notice that interstitials (as for example $\mathrm{Au}$ or $\mathrm{Fe}$ ) usually diffuse extremely rapidly. Therefore, in analogy to arguments given by Janzen et al., ${ }^{14}$ our results, that a certain fraction of interstitials is possible for $S$, can be interpreted to mean that $\mathrm{S}$ diffuses faster than $\mathrm{Se}$ and $\mathrm{Te}$. This interpretation is consistent with measurements which show that the diffusion coefficient decreases significantly from $S$ to Te, i.e., from $-10^{-8} \mathrm{~cm}^{2} / \mathrm{s}$ for $S$, to $-10^{-9} \mathrm{~cm}^{2} / \mathrm{s}$ for Se to $\sim 5 \times 10^{-12} \mathrm{~cm}^{2} / \mathrm{s}$ for Te (see Ref. 14, Fig. 3, at $1200^{\circ} \mathrm{C}$ and references therein, or Table 1 in Ref. 4).

In the total-energy calculations in Table I the distortions of the $\mathrm{Si}$ lattice were not taken into account. This effect was calculated independently by the selfconsistent pseudopotential method. ${ }^{21}$ We find that lattice relaxation would lower the energies by less than $0.3 \mathrm{eV}$ for the substitutional defects (see for example Fig. 5 in Ref. 20). For the $T_{d}$ interstitials the effect of lattice distortions is estimated to lower the total energy by less than $0.8 \mathrm{eV}$. As a consequence, the effect of lattice distortions is not important for the above arguments.

Additional support for the identification of the substitutional site is obtained by comparison of the theoretical electronic-structure results with available experimental data. In Fig. 1 we show the defectinduced changes in the density of states due to an interstitial and a substitutional Se impurity atom in $\mathrm{Si}$. For reasons of clarity only states of $A_{1}$ ( $s$-like at the impurity) and $T_{2}$ ( $p$-like at the impurity) symmetries are displayed. As might be expected from the elec- 


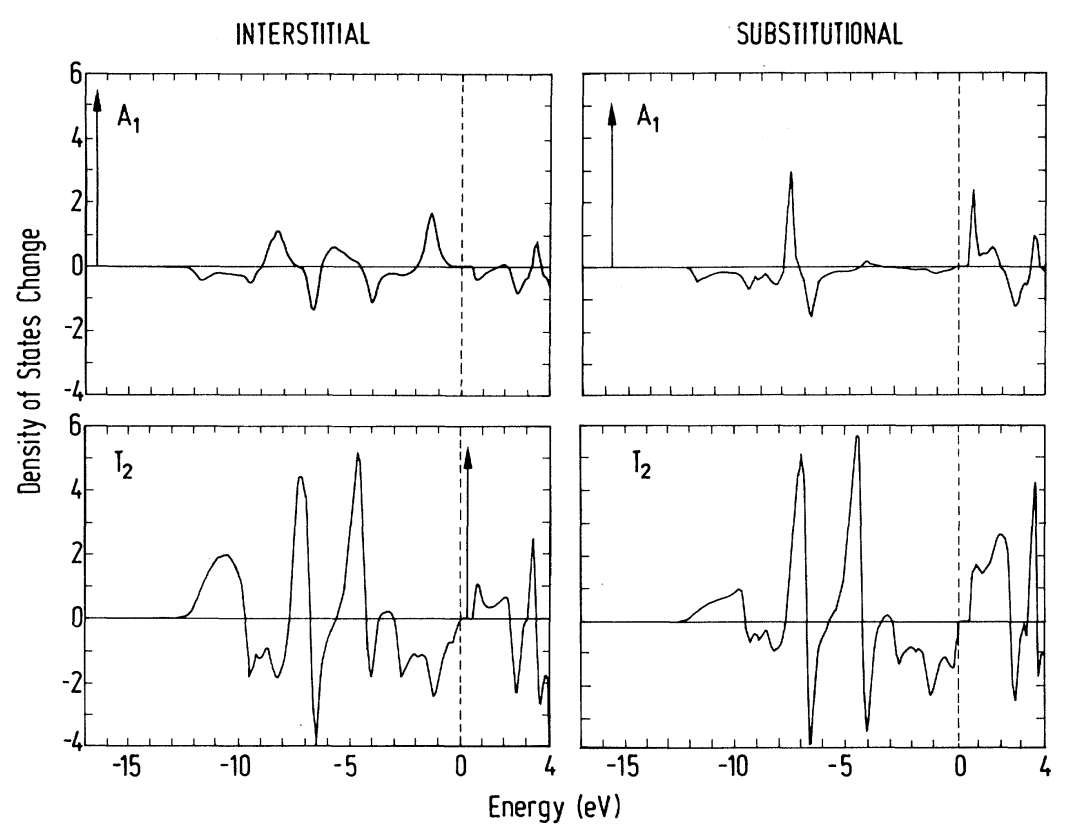

FIG. 1. The changes in the density of states of $A_{1}$ and $T_{2}$ symmetries induced by an interstitial (left) and a substitutional (right) Se atom in silicon. The energy zero is the top of the valence band.

tronic structure of the considered impurities (no $d$ electrons), the effects on states of other symmetries are less important. Qualitatively similar results are obtained for S and Te. The general effect visible in Fig. 1 is that states are shifted to lower energies. For the interstitial this arises because the potentials of these defects are attractive compared to the slightly repulsive potential in the interstitial region of the defect-free $\mathrm{Si}$ crystal. All undistorted $T_{d}$ interstitial chalcogens have a $T_{2}$ one-electron state in the gap, which in the neutral charge state is filled with four electrons. The site symmetry of paramagnetic chalcogen point defects was determined by infrared absorption experiments, ${ }^{4,5,9}$ EPR, ${ }^{9}$ and ENDOR $^{2,6}$ as $T_{d}$ and the spin of the paramagnetic state was found ${ }^{2,6,9}$ to be $\frac{1}{2}$. These experimental results are not consistent with the theoretical results for interstitial chalcogens: Here all manyelectron states with spin $\frac{1}{2}$ are orbital degenerate and therefore unstable with respect to a symmetry-lowering Jahn-Teller distortion. Thus, the site symmetry of these interstitials must be lower than $T_{d}$. Furthermore, infrared absorption as well as EPR and ENDOR show that the deep-level wave function has no node at the chalcogen site, thus transforming according to the $A_{1}$ representation. Again, this experimental result is in contradiction to the theoretical results for interstitial centers (see Fig. 1). It therefore can be ruled out that the experimental data arise from interstitial chalcogens.

For substitutional chalcogens we find again that states are shifted to lower energies (see Fig. 1). This can be explained by the higher electronegativity of chalcogens compared to that of a Si atom which is replaced. The unmodified LDA calculations do not give a level in the band gap, but there is a sharp $A_{1}$ resonance at the bottom of the conduction band. Since sulfur has two valence electrons more than $\mathrm{Si}$, we are only able to calculate the double-positive charge state and to put two electrons into the conduction band. If we correct for the band-gap problem (see above), the $A_{1}$ resonance becomes a bound state in the gap, in agreement with earlier pseudopotential Green's-function calculations ${ }^{28,29}$ and with the experimental results. $^{2,4-6,9}$

In conclusion, we have shown that the calculation of dissolution and reaction energies allows one to identify the stable configuration of defects in semiconductors. For isolated $\mathrm{S}, \mathrm{Se}$, and $\mathrm{Te}$ impurities in $\mathrm{Si}$ the theoretical results imply that the stable position of these atoms is the substitutional site. This identification is supported by a comparison of the calculated electronic structure with EPR, ENDOR, and infrared-absorption data. Whereas for Te the substitutional site has significantly lower energy than the interstitial site, our results indicate that for $\mathrm{S}$ a certain amount of interstitials is possible if Si self-interstitials are present.

We are grateful to E. Weber, who pointed out to us the experimental diffusion data. We thank him, as well as O. K. Andersen and W. Frank for helpful discussions. 
1M. Lannoo and J. Bourgoin, Point Defects in Semiconductors I (Springer-Verlag, Berlin, 1981).

${ }^{2}$ G. W. Ludwig, Phys. Rev. A 37, 1520 (1965).

${ }^{3}$ B. K. Meyer, J. M. Spaeth, and M. Scheffler, Phys. Rev. Lett. 52, 851 (1984).

4P. Wagner, C. Holm, E. Sirtl, R. Oeder, and W. Zulehner, in Festkörperprobleme: Advances in Solid State Physics, edited by P. Grosse (Vieweg, Braunschweig, 1984), Vol. XXIV, p. 191.

${ }^{5}$ E. Janzen, R. Stedmann, G. Grossmann, and H. G. Grimmeiss, Phys. Rev. B 29, 1907 (1984).

6J. R. Niklas and J. M. Spaeth, Solid State Commun. 46, 121 (1983), and private communications.

${ }^{7}$ H. G. Grimmeiss, E. Janzen, and B. Skarstam, J. Appl. Phys. 51, 3740 (1980).

${ }^{8}$ C. Hoffmann and M. Schulz, Appl. Phys. A 33, 19 (1981).

${ }^{9}$ H. G. Grimmeiss, E. Janzen, H. Ennen, O. Schirmer, J. Schneider, R. Wörner, C. Holm, E. Sirtl, and P. Wagner, Phys. Rev. B 24, 4571 (1981).

10J. Dietl, D. Helmreich, and E. Sirtl, in Crystals: Growth, Properties, and Applications (Springer, Berlin, 1981), Vol. 5, p. 43.

11S. D. Brotherton, M. J. King, and G. J. Parker, J. Appl. Phys. 52, 4649 (1981).

${ }_{12}$ A. L. Lin, A. G. Crouse, J. Wendt, A. G. Campbell, and R. Newman, Appl. Phys. Lett. 38, 683 (1981).

13O. F. Schirmer, Physica (Utrecht) 116B, 306 (1982).

${ }^{14}$ E. Janzen, H. G. Grimmeiss, A. Lodding, and Ch. Deline, J. Appl. Phys. 53, 7367 (1982).

15J. Bernholc, N. O. Lipari, and S. T. Pantelides, Phys. Rev. B 18, 1780 (1980).

${ }^{16}$ G. A. Baraff and M. Schlüter, Phys. Rev. B 19, 1965 (1979).

${ }^{17}$ O. Gunnarsson, O. Jepsen, and O. K. Andersen, Phys. Rev. B 27, 7144 (1983).

${ }^{18}$ R. Car, P. J. Kelly, A. Oshiyama, and S. T. Pantelides,
Phys. Rev. Lett. 52, 1814 (1984).

${ }^{19} \mathrm{G}$. Baraff and M. Schlüter, Phys. Rev. B 30, 3460 (1984).

${ }^{20} \mathrm{M}$. Scheffler, F. Beeler, O. Jepsen, O. Gunnarsson, O. K. Andersen, and G. B. Bachelet, J. Electron. Mater. 14A, 45 (1985).

${ }^{21}$ M. Scheffler, J. P. Vigneron, and G. B. Bachelet, Phys. Rev. Lett. 49, 1765 (1982); Phys. Rev. B 31, 6541 (1985).

${ }^{22}$ F. Beeler, O. Jepsen, O. Gunnarsson, O. K. Andersen, and $\mathrm{M}$. Scheffler, to be published.

23J. P. Perdew and M. Levy, Phys. Rev. Lett. 51, 1884 (1983).

${ }^{24}$ L. J. Sham and M. Schlüter, Phys. Rev. Lett. 51, 1888 (1983).

${ }^{25} \mathrm{We}$ use the following definition of the scissor operator: $\tilde{G}^{0}(E)$ (Ref. 17) is calculated with use of a rigid shift of the conduction-band density of states, as by Baraff and Schlüter (Ref. 19). The corresponding potential function $\tilde{P}^{0}(E)$ (Ref. 17) is not modified.

${ }^{26}$ Of the methods for correcting the band gap, which we have tested, the scissor operator (Ref. 19) has the most dramatic effect on the total energy of the donor defect system. A rigid shift $\Delta$ of the conduction band increases the total energy by $\Delta E \geqslant M \Delta$, where $M$ is the defect-induced increase in the number of occupied states (which, by Levinson's theorem, are derived from the conduction band). This argument implies that the effect is drastically different for an all-electron potential and a pseudopotential or frozen-core approximation. For interstitial chalcogens in the frozen-core approximation $\Delta E \geqslant 6 \times 0.6 \mathrm{eV}=3.6 \mathrm{eV}$ (see also Ref. 22).

${ }^{27}$ The results of Table I for $E^{\text {sub }}$ differ from those given in Ref. 20 because of a different treatment of the band-gap problem (see also Ref. 22).

28J. Bernholc, N. O. Lipari, S. T. Pantelides, and M. Scheffler, Phys. Rev. B 26, 5706 (1982).

${ }^{29}$ V. A. Singh, U. Lindefelt, and A. Zunger, Phys. Rev. B 27, 4909 (1983). 